\title{
THE SOUTHERLY AND EASTERLY DEVIATIONS OF FALLING
}

\section{BODIES FOR AN UNSYMMETRICAL GRAVITATIONAL}

\section{FIELD OF FORCE*}

BY

\section{WILLIAM H. ROEVER}

\section{Introduction and Statement of Results.}

The adequacy with which theory may be made to account for a given class of physical phenomena depends largely upon the nature of the assumptions which are made in the mathematical formulation of the corresponding physical problem. This is well illustrated in the problem of the southerly deviation of falling bodies. In the treatment of this problem (and that of the easterly deviation) by Gauss, the assumption is made that- the force in the statical field of force which is at rest with respect to the earth, i. e., the field of force in which the plumb-line is in equilibrium, is constant in magnitude and direction in the neighborhood of the path of the falling body. Under this assumption, $\dagger$ it follows from the laws of dynamics that the southerly deviation (denoted by S. D.) is given by the formula:‡

$$
\text { S. D. }=\frac{1}{3} \omega^{2} \sin 2 \phi \cdot \frac{h^{2}}{g},
$$

in which $\omega$ is the angular velocity of the earth's rotation, $h$ is the height through which the body falls, and $g$ and $\phi$ are the acceleration due to weight and the astronomical latitude, respectively, at the place of observation. In a recent paper by the author $\S$ it is shown that, under the assumption of a distribution of revolution (i. e., that the potential function of the earth's gravitational (or weight) field of force is of the form $f(r, z)$, where $r$ is the distance of a general point from the earth's axis of rotation, and $z$ is that from a fixed plane perpendicular to the axis), the same theory yields for the southerly deviation

\footnotetext{
* Presented to the Society December 2, 1911 (Southwestern Section) and April 5, 1912 (Chicago Section).

t Other assumptions are also made. The body is assumed to be a particle, and the effects of uir currents, air resistance and the actions of the moon and sun are disregarded.

‡ See Gavss's Werke, vol. 5 (1867), p. 502.

These Transactions, vol. 12 (1911), pp. 335-353.

Trans. Amer. Math. Soc. 80 
the formula

$$
\text { S. D. }=\frac{1}{6}\left[2 \omega^{2} \sin 2 \phi+5\left(\frac{\partial g}{\partial x}\right)_{0}\right] \frac{h^{2}}{g_{0}},
$$

in which $\partial g / \partial x$ is the derivative of $g$ along the meridian measured to the north, the subscript $(0)$ denoting the particular value at the initial point $P_{0}$ of the falling body. It will be observed that when $g$ is constant, $\partial g / \partial x=0$, and therefore formula (1) is a special case of formula (2).

For the potential function for which the Besselian ellipsoid is a level surface and the formula of Helmert gives the acceleration due to weight on this ellipsoid, the following table gives a few of the corresponding values of $\phi$, $g$, and $\partial g / \partial x .^{*}$

TABLE I.

\begin{tabular}{|c|c|c|}
\hline$\phi$ & $g$ & $+10^{\circ} \frac{\partial g}{\partial x}$ \\
\hline $40^{\circ}$ & 980.1457 & 8.0399 \\
$45^{\circ}$ & 980.5966 & 8.1568 \\
$50^{\circ}$ & 981.0475 & 8.0259 \\
$55^{\circ}$ & 981.4847 & 7.6517 \\
$60^{\circ}$ & 981.8949 & 7.0463 \\
\hline
\end{tabular}

On the other hand,

$$
\omega^{2}=5.3173 \cdot 10^{-9} .
$$

The values given by formulas (1) and (2) are proportional to

$$
2 \omega^{2} \sin 2 \phi \quad \text { and } 2 \omega^{2} \sin 2 \phi+5(\partial g / \partial x)_{0} .
$$

Since $\sin 2 \phi$ never exceeds unity, it is evident from Table I, that, for the

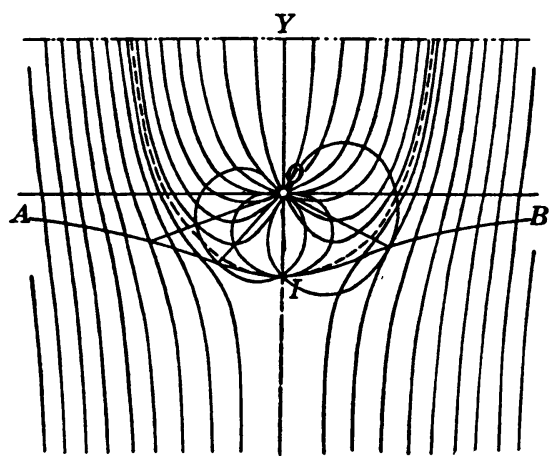

potential function for which the standard ellipsoid is a level surface, formula (2) gives values for the southerly deviation which are nearly five times as great as those given by formula (1).

The expression $\left(\partial g /_{0}^{0} d x\right)_{0} / g_{0}$ is the curvature, at $P_{0}$, of the line of force which passes through $P_{0}$ of the statical field of force which is at rest with respect to the earth. Thus we see that the curvature of these lines of force influences

* See 2 nd reference in the second fclloring footnote. 
more strongly the southerly deviation of falling bodies than does the earth's rotation.

Simple considerations show that the introduction of an attracting mass into a field of constant force, or into one whose lines of force have slight curvature, increases considerably the curvature of the lines of force in the neighborhood of the introduced mass.*

This fact suggests that the proximity, to an experiment station, of a mountain or of a mineral deposit can change considerably the curvature of the lines of force of the statical field which is at rest with respect to the earth, and thus affect considerably the southerly deviation of falling bodies. But the proximity of such a disturbing factor would make the distribution of matter which produces the earth's gravitational field of force cease to be one of revolution. Therefore it becomes necessary to derive a formula for the southerly deviation-one for the easterly deviation will also be derived-under the assumption of a distribution not of revolution. This has been done in this paper. (See formulas III, where $\bar{\xi}=$ S. D. and $\bar{\eta}=$ E. D.) It turns out that the first term of the expression for the southerly deviation, under this assumption, is the same as expression (2), and that the term in $h^{5 / 2}$, which does not appear when the assumption of a distribution of revolution is made, is negligible in comparison with the first term.

The Hungarian physicist Baron Roland Eötvös has recently devised a method by means of which he can determine experimentally the derivative $\partial g / \partial x$, and also other second derivatives of the potential function $W$ of the force due to weight. $\dagger$ The following table gives the observed values of these derivatives at a few stations which are about twenty kilometers east of Arad in Hungary and just west of some mountains.

TABLE II.

\begin{tabular}{|c|c|c|c|c|}
\hline Station number & $10^{\circ} \frac{\partial^{2} W}{\partial x \partial z}$ & $10^{\circ} \frac{\partial^{2} W}{\partial y \partial z}$ & $10^{\circ}\left(\frac{\partial^{2} W}{\partial y^{3}}-\frac{\partial^{2} W}{\partial x^{2}}\right)$ & $10^{\circ} \frac{\partial^{2} W}{\partial x \partial y}$ \\
\cline { 1 - 4 } & +32.9 & +88.1 & +37.0 & +3.8 \\
\cline { 1 - 4 } 1,018 & +25.8 & +62.8 & +72.8 & +2.5 \\
1,032 & +15.5 & +69.8 & +25.5 & +4.6 \\
2,159 & +37.0 & +34.5 & +34.3 & +13.5 \\
2,188 & +77.8 & +59.8 & +21.5 & +30.3 \\
2,192 & +82.3 & +64.5 & +28.2 & +26.7 \\
\hline
\end{tabular}

* The opposite figure represents the lines of force of the gravitational field which is produced by the introduction of a mass $m$, at 0 , into a field which originally was constant (and of which the lines of force were straight lines parallel to $O Y$ ). The line $A I B$ is the locus along which the curvature has not been changed; in this case, it is the locus of the points of inflection of the lines of force of the new field of force.

† See Encyklopädieder mathematischen Wissenschaften, Band VIrB, \$23, p. 166. Also, Verhandlungender fünfzehnten allgemeinen Conferenzderinternationalen Erdmessung, 1 (1906), pp. 337-395. 
Here $x$ is measured to the north, $y$ to the east, and $z$ downward along the vertical. Hence

$$
g=\frac{\partial W}{\partial z}, \quad \frac{\partial g}{\partial x}=\frac{\partial^{2} W}{\partial x \partial z}, \quad \frac{\partial g}{\partial y}=\frac{\partial^{2} W}{\partial y \partial z} .
$$

For the latitude $\left(46^{\circ} 10^{\prime}\right)$ of this region, the normal values (i. e., the values corresponding to the potential function for which the standard ellipsoid is a level surface) of the quantities given in Table II are

respectively.

$$
8.1, \quad 0, \quad 4.9, \quad 0,
$$

At station 2,192 the value of the expression $2 \omega^{2} \sin 2 \phi+5(\partial g / \partial x)_{0}$ is about $422 \cdot 10^{-9}$, and hence formula (2) gives a value forty trmes as great as formula (1). On the other hand, at station $2,159,2 \omega^{2} \sin 2 \phi+5(\partial g / \partial x)_{0}$ is $-174 \cdot 10^{-9}$, and hence formula (2) gives a northerly devation which is more than sixteen times as great as the southerly deviation given by formula (1) The local value of the derivative $\partial g / \partial x=\partial^{2} W / \partial x \partial z$ thus appears to determine the extent and the sign of the southerly deviation of falling bodies.

The easterly deviation of falling bodies is also influenced by the proximity of mountains or of mineral deposits. In fact, such disturbing factors yield the second term of the expression for the easterly deviation (denoted by E. D.). See formulas III.

$$
\text { E. D. }=\frac{2}{3} \sqrt{2} \omega \cos \phi \frac{h^{3^{/ 2}}}{g_{0}^{1 / 2}}-\frac{5}{6} \frac{(\partial g / \partial y)_{0}}{g_{0}} h^{2} \text {. }
$$

From Table II and the form of the second term in formula (3), it is evident that the effect of such disturbing factors is comparable in magnitude to the southerly deviation, but small in comparison with the first term of formula (3). The effect is also comparable in magnitude to the effect which Gauss finds is due to air resistance. Thus, for the data of Benzenberg's experiment in St. Michael's Tower, namely $\phi=53^{\circ} 33^{\prime}$ and $h=235$ feet, the formula of Gauss for the easterly deviation (which is equivalent to the first term of formula (3)) gives the value 3.91 lines. ${ }^{*}$ When air resistance is taken into account, Gauss finds for the same data the deviation 3.86 lines. Hence the effect of air resistance is -.05 lines. On the other hand the value of the second term of formula (3) for the data of station 1018 (Table II) is - .02 lines. Gauss states that for the southerly deviation the effect of air resistance is negligible.

In this paper the effects of air resistance, air currents, and the actions of the moon and sun have not been taken into consideration. $\dagger$ While the com-

$* 144$ lines $=1$ foot.

† Another matter which has not been taken into consideration in this paper is the weight of the string which supports the plumb-bob. In a field of force in which the lines of force are not rectilinear, this string will have some curvature. Therefore the position of a plumb-bob which is supported by a string with weight will be slightly different from that of a bob which is supported by a weightless string of the same length as the heavy string. 
bined effects of these influences may be appreciable, it seems desirable to do first what has been done in this paper, namely to determine the effect of a local irregularity in the earth's gravitational field of force. The surprising conclusion is reached that known local irregularttes in the earth's gravtational field of force (caused by the presence of mountains or of mineral deposits or even by large bunldings or tunnels) (1) influence the southerly deviation to the extent of from -16 times to +40 trmes* the amount whrch 28 given by the formula of Gauss for the southerly deviation (formula (1)), (2) affect the easterly devation by amounts which are comparable with the effect which Gauss finds is due to air resustance.

\section{\$1. Defintions of, and formulas for, the easterly and southerly devations of falling bodres.}

In experiments for the determination of the easterly and southerly deviations of falling bodies, a plumb-line $P_{0} R$ is supported at the point $P_{0}$ from which a spherical body is later permitted to fall. On the horizontal plane which passes through the plumb-bob $R$, the easterly and southerly directions $R E_{R}$ and $R S_{R}$ are drawn (Fig. 3). The plumb-line $R P_{0}$ is the vertical at $R . \dagger$ The falling body, after being released from the position $P_{0}$, moves in a path which, with respect to the axes $R-E_{R}, S_{R}, P_{0}$, is the curve $c$. This curve pierces the horizontal plane $E_{R} R S_{R}$ in the point $C$. The distance by which $C$ lies to the south of $R E_{R}$ is called the southerly devation of the fallng body, and that by whrch it lies to the east of $R S_{R}$ is called the easterly devation.

In order to get expressions for these deviations the curve $c$ is referred to the axes $P_{0}-\bar{\eta}, \bar{\xi}, \bar{\xi}$ which pass through $P_{0}$ and are parallel to the axes $R-E_{R}, S_{R}, P_{0}$. The equations of the curve $c$ are (by Eqs. (17) $\S 7$ )

$$
\begin{aligned}
\bar{\eta}=-W_{\zeta}^{0}\left\{\frac{1}{3} \omega \cos \phi\right. & \cdot t^{3}+\frac{5}{24} W_{\eta \zeta}^{0} \cdot t^{4} \\
& \left.+\frac{1}{60} \omega\left(\sin \phi W_{\zeta \zeta}^{0}+\cos \phi\left(W_{\zeta \zeta}^{0}+W_{\eta \eta}^{0}-4 \omega^{2}\right)\right) t^{5}\right\},
\end{aligned}
$$

I. $\xi=-W_{\zeta}^{0}\left\{\frac{1}{24}\left(4 \omega^{2} \sin \phi \cos \phi+5 W_{\xi \xi}^{0}\right) t^{4}\right.$

$$
\begin{array}{r}
\left.+\frac{1}{60} \omega\left(9 \sin \phi W_{\eta \zeta}^{0}+\cos \phi W_{\xi \eta}^{0}\right) t^{5}\right\}, \\
\bar{\zeta}=\frac{1}{2} W_{\zeta}^{0}\left\{t^{2}+\frac{1}{12}\left(W_{\zeta \zeta}^{0}-4 \omega^{2} \cos ^{2} \phi\right) t^{4}-\frac{1}{3} \omega \cos \phi \cdot W_{\eta \zeta}^{0} \cdot t^{5}+\cdots\right\},
\end{array}
$$

* In the region of the Alps this range of values would probably be much greater. See note by M. Brillouin on the curvature of the geoid in the Simplon tunnel, C o m p t e s r e n d u s, vol. 102 (1906), p. 916.

$\dagger$ It is assumed that the plumb-bob is a heavy particle and that the line is weightless and perfectly flexible.

$\ddagger$ The axes $P_{0}-\bar{\eta}, \bar{\xi}, \bar{\zeta}$ do not coincide with the axes $P_{0}-\eta, \xi, \zeta$. The latter axes are the easterly, southerly and vertical directions at $P_{\mathrm{c}}$ 
where $t$ represents the time measured from the instant the body begins to fall, $\omega$ represents the angular velocity of the earth's rotation, $\phi$ the latitude of the point $P_{0}, W$ the potential function of the statical field of force which is at rest with respect to the rotating earth, and the symbols $W_{\zeta}^{0}, W_{\eta \zeta}^{0}, W_{\xi \zeta}^{0}$, $W_{\zeta \zeta}^{0}, W_{\eta \eta}^{0}, W_{\xi \eta}^{0}$, stand for the values of the derivatives

$$
\frac{\partial W}{\partial \zeta}, \frac{\partial^{2} W}{\partial \eta \partial \zeta}, \frac{\partial^{2} W}{\partial \xi \partial \zeta}, \frac{\partial^{2} W}{\partial \zeta^{2}}, \frac{\partial^{2} W}{\partial^{2} \eta}, \frac{\partial^{2} W}{\partial \xi \partial \eta}
$$

at the point $P_{0}$. If we put $-\zeta=h=R P_{0}$, where $h$ is the height through which the body falls, the corresponding values of $\bar{\eta}$ and $\bar{\xi}$ are respectively the easterly and the southerly deviations which correspond to $h$.

Let us now express $\bar{\eta}$ and $\bar{\xi}$ explicitly in terms of $h$. The last equation assumes the form

$$
\frac{h}{\alpha}=t^{2}+\gamma t^{4}+\delta t^{5}+\cdots
$$

where

$$
\alpha=-\frac{1}{2} W_{\zeta}^{0}, \quad \gamma=\frac{1}{12}\left(W_{\zeta \zeta}^{0}-4 \omega^{2} \cos ^{2} \phi\right), \quad \delta=-\frac{1}{3} \omega \cos \phi \cdot W_{\eta \zeta}^{0} .
$$

Hence

$$
\begin{aligned}
\pm \sqrt{\frac{h}{\alpha}} & =\sqrt{t^{2}+\gamma t^{4}+\delta t^{5}+\cdots} \\
& =t+\frac{1}{2} \gamma t^{3}+\frac{1}{2} \delta t^{4}+\cdots
\end{aligned}
$$

and this when solved for $t$ yields the relation

$$
t=\left( \pm \sqrt{\frac{h}{\alpha}}\right)-\frac{1}{2} \gamma\left( \pm \sqrt{\frac{h}{\alpha}}\right)^{3}-\frac{1}{2} \delta\left( \pm \sqrt{\frac{h}{\alpha}}\right)^{4}+\cdots
$$

Since $t$ is positive, the upper sign must be used. The expressions for $\eta$ and $\bar{\xi}$ then assume the forms:

$$
\begin{aligned}
\bar{\eta}= & \frac{2}{3} \sqrt{2} \cdot \omega \cos \phi \cdot \frac{h^{3 / 2}}{\sqrt{-W_{\zeta}^{0}}}+\frac{5}{6} \frac{W_{\eta \xi}^{0}}{\left(-W_{\zeta}^{0}\right)} h^{2} \\
& +\frac{\sqrt{2}}{30} \omega\left(2 \sin \phi \cdot W_{\xi \zeta}^{0}+2 \cos \phi \cdot W_{\eta \eta}^{0}-3 \cos \phi \cdot W_{\zeta \zeta}^{0}\right.
\end{aligned}
$$

and the expression for $\bar{\zeta}$ assumes the form $\bar{\zeta}=-h$, as it should. Since 
$W_{\eta}, W_{\xi}, W_{\zeta}$ are the rectangular components of the acceleration $g$ due to weight, we may write

$-W_{\zeta}^{0}=g_{0}, \quad W_{\eta \zeta}^{0}=-\left(\frac{\partial g}{\partial \eta}\right)_{0}, \quad W_{\xi \zeta}^{0}=-\left(\frac{\partial g}{\partial \xi}\right)_{0}, \quad W_{\zeta \zeta}^{0}=-\left(\frac{\partial g}{\partial \zeta}\right)_{0}$,

and therefore the above formulas may be written as follows:

$$
\begin{aligned}
& \bar{\eta}= \frac{2}{3} \sqrt{2} \cdot \omega \cos \phi \cdot \frac{h^{3 / 2}}{g_{0}^{1 / 2}}-\frac{5}{6} \frac{(\partial g / \partial \eta)_{0}}{g_{0}} h^{2} \\
&+\frac{\sqrt{2}}{30} \omega\left[3 \cos \phi\left(\frac{\partial g}{\partial \zeta}\right)_{0}-2 \sin \phi\left(\frac{\partial g}{\partial \xi}\right)_{0}+2 \cos \phi\left(\frac{\partial W_{\eta}}{\partial \eta}\right)_{0}\right. \\
&\left.+4 \omega^{2}\left(5 \cos ^{3} \phi-2 \cos ^{2} \phi\right)\right] \frac{h^{5 / 2}}{g_{0}^{3 / 2}}
\end{aligned}
$$

III.

For a distribution of revolution we have

$$
\frac{\partial^{2} W}{\partial \eta \partial \zeta}=0, \quad \frac{\partial^{2} W}{\partial \eta \partial \xi}=0,
$$

and therefore the term in $h^{2}$ in the expression $(\bar{\eta})$ for the easterly deviation and the term in $h^{5 / 2}$ in expression $(\bar{\xi})$ for the southerly deviation drop out.

\section{The establishment of the general equations $I$.}

$\S 2$. The curve'c. The curve $c$ has already been defined as the path of the falling particle with respect to the rotating earth. Let us first refer it to a set of rectangular axes $O-x, y, z$, fixed in the rotating earth, and such that the origin $O$ is a fixed point (interior to the earth) of the earth's axis $a$ of rotation. $O z$ is coincident with $a$, and positive in the direction of the north pole, $O x$ is perpendicular to $O z$ and is so chosen that the initial point $P_{0}$ lies in the plane $z O x$, the positive direction being that from $O z$ to $P_{0}$, and finally $O y$ is perpendicular to $O z$ and $O x$ and is positive in the direction which $O x$ would have if it were revolved around the axis $O z$ through an angle of $90^{\circ}$ in the direction of the earth's rotation. (See Fig. 1.)

If we represent by $\omega$ the angular velocity of the earth's rotation, and by $U$ the function $f(x, y, z)$ which is defined by the integral:

$$
U=\int_{V} \frac{d m}{\rho}, \quad \rho=\sqrt{\left(x-x_{i}\right)^{2}+\left(y-y_{i}\right)^{2}+\left(z-z_{i}\right)^{2}},
$$

where $d m$ represents that element of mass of the earth which is situated at 
the point whose coordinates, with respect to the axes $O-x, y, z$, are $x_{i}, y_{i}, z_{i}$, the integration being extended throughout the whole volume of the earth, then the function:

$$
W=U+\frac{\omega^{2}}{2}\left(x^{2}+y^{2}\right)
$$

is the potential function of the statical field of force which is at rest with respect to the rotating earth* (i. e., the lines of force:

$$
d x: d y: d z=\frac{\partial W}{\partial x}: \frac{\partial W}{\partial y}: \frac{\partial W}{\partial z}
$$

of this field are at rest with respect to the rotating earth $\dagger$ ).

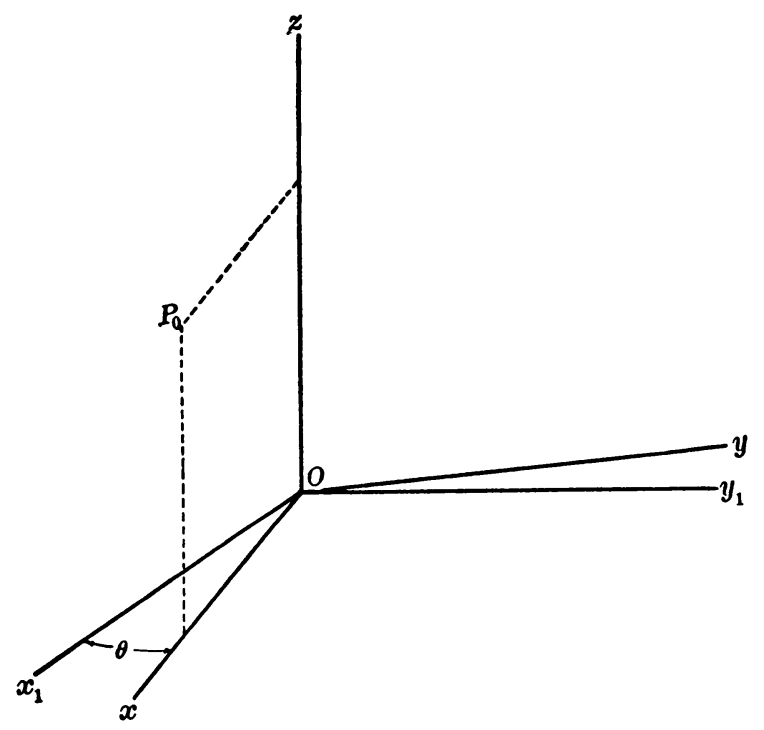

Fig. 1.

In order to find the differential equations of motion, let us denote by $O-x_{1}, y_{1}, z$ a set of rectangular axes which are fixed in space and so oriented that at the instant $t=0$, when a falling particle leaves $P_{0}$ (at rest with respect to the earth), the set of axes $O-x, y, z$ coincides with the set $O-x_{1}, y_{1}, z$ (see Fig. 1). If we denote by $\theta$ the angle $x_{1} O x$, positive in the direction in which the earth rotates, the relations between the coordinates are: Then

$$
x=x_{1} \cos \theta+y_{1} \sin \theta, \quad y=-x_{1} \sin \theta+y_{1} \cos \theta, \quad z=z_{1} .
$$

$$
U=f(x, y, z)=f_{1}\left(x_{1}, y_{1}, z, t\right),
$$

* See Pizzerti, Tratlato di Geodesia teoretica, $\$ 2$ (1905).

† It should be borne in mind that the level (equipotential) surfaces, $W=$ const., are not necessarily surfaces of revolution. 
where $\theta=\omega t$ is a function of the time $t$. The differential equations of motion with respect to the fixed axes $O-x_{1}, y_{1}, z$, are

$$
\frac{d^{2} x_{1}}{d t^{2}}=\frac{\partial U}{d x_{1}}, \quad \frac{d^{2} y_{1}}{d t^{2}}=\frac{\partial U}{\partial y_{1}}, \quad \frac{\partial^{2} z}{d t^{2}}=\frac{\partial U}{\partial z} .
$$

If we subject these differential equations to the inverse of the transformation just given, namely to the transformation

$$
x_{1}=x \cos \theta-y \sin \theta, \quad y_{1}=x \sin \theta+y \cos \theta, \quad z_{1}=z,
$$

we obtain a set of differential equations* which is equivalent to the set:

$$
\frac{d^{2} x}{d t^{2}}-2 \omega \frac{d y}{d t}-\omega^{2} x=\frac{\partial U}{\partial x}, \quad \frac{d^{2} y}{d t^{2}}+2 \omega \frac{d x}{d t}-\omega^{2} y=\frac{\partial U}{\partial y}, \quad \frac{d^{2} z}{d t^{2}}=\frac{\partial U}{\partial z} .
$$

Since by equation (1),

$$
\frac{\partial U}{\partial x}+\omega x=\frac{\partial W}{\partial x}, \quad \frac{\partial U}{\partial y}+\omega y=\frac{\partial W}{\partial y}, \quad \frac{\partial U}{\partial z}=\frac{\partial W}{\partial z},
$$

these equations assume the forms:

$$
\frac{d^{2} x}{d t^{2}}-2 \omega \frac{d y}{d t}=\frac{\partial W}{\partial x}, \quad \frac{d^{2} y}{d t^{2}}+2 \omega \frac{d x}{d t}=\frac{\partial W}{\partial y}, \quad \frac{d^{2} z}{d t^{2}}=\frac{\partial W}{\partial z} .
$$

The curve $c$ is then that solution of the differential equations (2) which is subject to the initial conditions:

(3) When $t=0, \quad x=r_{0}, \quad y=0, \quad z=z_{0}, \quad \frac{d x}{d t}=\frac{d y}{d t}=\frac{d z}{d t}=0$.

§3. The curve $c$ referred to the cardinal axes at $P_{0}$. Before we can define the cardinal axes it will be necessary to define the following terms. At a general point $P$ which is at rest with respect to the rotating earth, the vertical is the straight line which coincides with the plumb-line, $\dagger$ the plumb-bob of

$$
\begin{aligned}
& d^{2} x \cos \theta-\frac{d^{2} y}{d t^{2}} \sin \theta-2\left(\frac{d x}{d t} \sin \theta+\frac{d y}{d t} \cos \theta\right) \omega-(x \cos \theta-y \sin \theta) \omega^{2} \\
&= \frac{\partial U}{\partial x} \cos \theta-\frac{\partial U}{\partial y} \sin \theta \\
& \frac{d^{2} x}{d t^{2}} \sin \theta+\frac{d^{2} y}{d t^{2}} \cos \theta+2\left(\frac{d x}{d t} \cos \theta-\frac{d y}{d t} \sin \theta\right) \omega-(x \sin \theta+y \cos \theta) \omega^{2}=\frac{\partial U}{\partial x} \sin \theta+\frac{\partial U}{\partial y} \cos \theta
\end{aligned}
$$

If we multiply the first of these equations by $\cos \theta$ and the second by $\sin \theta$ and add, we obtain

$$
\frac{d^{2} x}{d t^{2}}-2 \omega \frac{d y}{d t}-\omega^{2} x=\frac{\partial U}{\partial x}
$$

and if we multiply the first by $-\sin \theta$ and the second by $\cos \theta$ and add, we obtain

$$
\frac{d^{2} y}{d t^{2}}+2 \omega \frac{d x}{d t}-\omega^{2} y=\frac{\partial U}{\partial y} \text {. }
$$

it is assumed that the plumb-bob is a heavy particle and that the line is weightless and perfectly flexible. 
which is situated at $P$ (this line does not necessarily intersect the axis of rotation $a$ of the earth), the meridian plane is the plane which passes through the vertical and is parallel to the axis $a$, the horizontal plane is the plane which passes through $P$ and is perpendicular to the vertical, the east-and-west line is the straight line which passes through $P$ and is perpendicular to the meridian plane, the north-and-south line is the line of intersection of the meridian and the horizontal planes. By the cardinal axes at the point $P$ we shall mean the vertical, the east-and-west and the north-and-south lines at the point $P$.

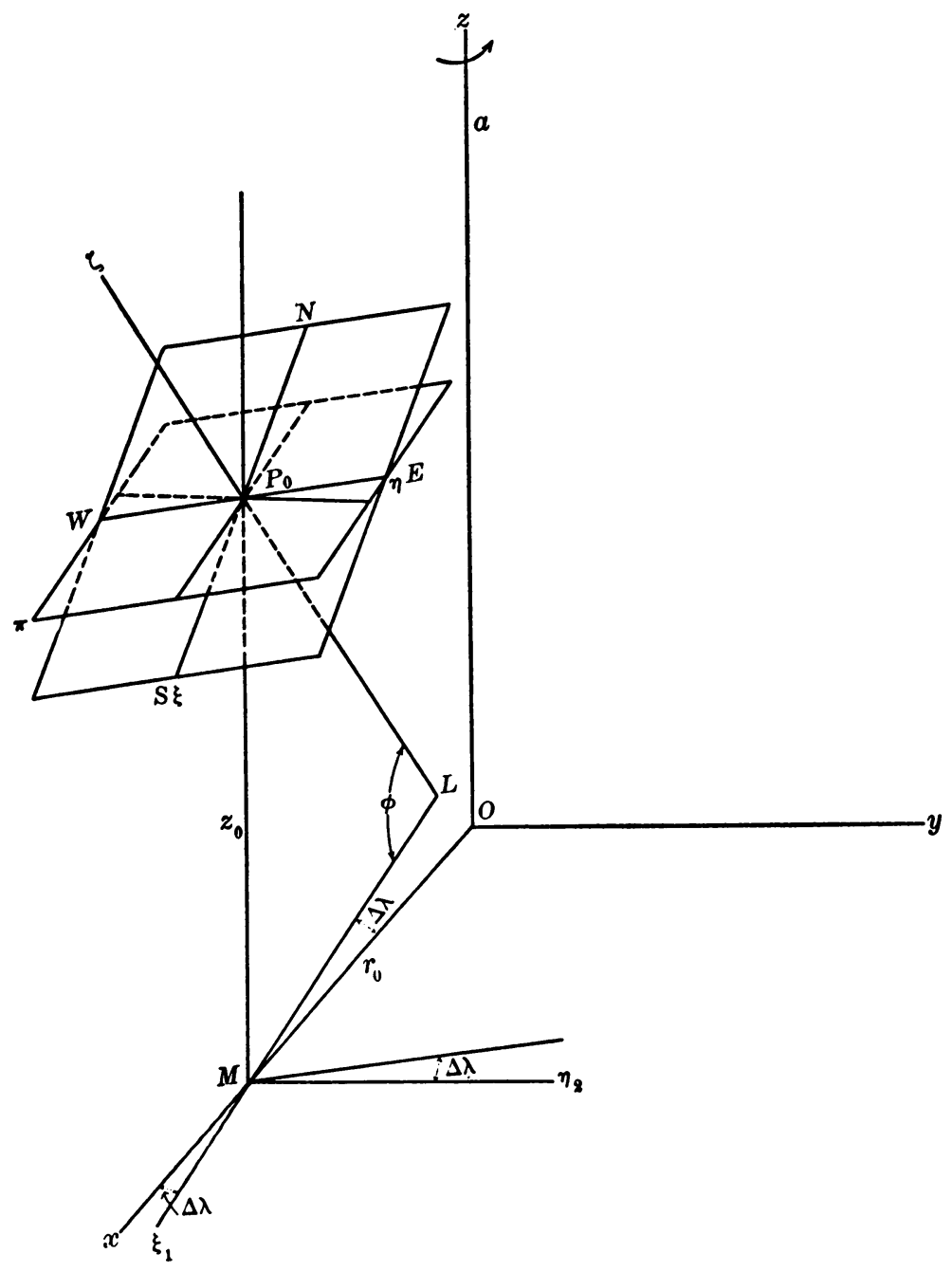

Frg. 2.

The positive directions of these axes shall be those toward the zenith, the east, and the south respectively. A few more terms will now be defined. The 
latitude at $P$ is the complement of the angle which the positive direction of the vertical at $P$ makes with the positive direction of the axis $a$. The longitude at $P$ is the angle which the meridian plane at $P$ makes with a fixed plane through the axis $a$ (we shall here assume that the fixed plane is the plane $z O x$ ) measured from $0^{\circ}$ to $360^{\circ}$ to the east.*

Let us now take the cardinal axes at the point $P_{0}$ as the axes of the coordinates $\xi, \eta, \zeta$, where $\xi$ is measured along the north-and-south line, $\eta$ along the east-and-west line and $\zeta$ along the vertical. See Fig. 2, in which $O z \equiv a$ represents the axes of rotation of the earth, and $O x, O y$ a pair of

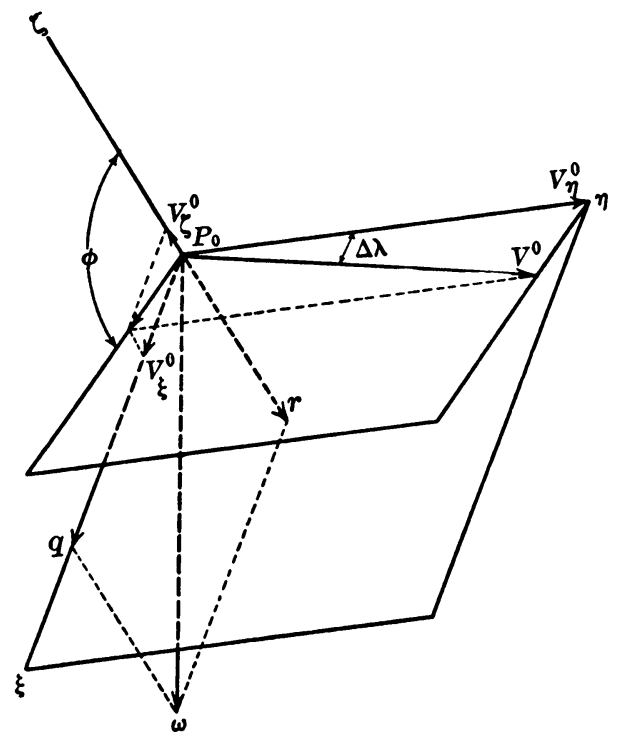

Fig. ${ }^{\prime}$.

rectangular axes which are at rest with respect to the rotating earth and are perpendicular to $O z$ at $O . P_{0}$ is a point of the plane $x O z . \quad P_{0} M$ is parallel to $a$, and intersects the plane $x O y$ in $M$. We put

$$
r_{0}=O M, \quad z_{0}=M P_{0} .
$$

The line $P_{0} L$ is the vertical at $P_{0}$; it intersects the plane $x O y$ in $L . L P_{0} M$ is then the meridian plane at $P_{0}$. The plane $N E S W$, which passes through $P_{0}$ and is perpendicular to $P_{0} L$, is the horizontal plane at $P_{0}$. These two planes intersect in the north-and-south-line NS. Let us denote by $\pi$ the plane which passes through $P_{0}$ and is aparallel to the plane $x O y$. This plane intersects the horizontal plane in the east-and-west line $E W$.

We agreed to take the plane $x O z$ as the plane from which to measure longitude. Therefore the longitude of $P_{0}$ is the angle $\Delta \lambda=\Varangle O M L$. The latitude of $P_{0}$ is the angle $\phi=\Varangle M L P_{0}$.

\footnotetext{
* See Pizzetri, loc. cit., $\S 5$.
} 
In order to get the equations of transformation from the axes $O-x, y, z$ to the axes $P_{0}-\xi, \eta, \zeta$, let us introduce the auxiliary variables $\xi_{1}, \zeta_{1}, \xi_{2}, \eta_{2}$, where $\xi_{1}$ represents distance measured along $M L$ from $M$ in the direction $L M$, and $\xi_{1}, \xi_{2}$ and $\eta_{2}$ are defined by the equations

Then

$$
x=\xi_{2}+r_{0}, \quad y=\eta_{2}, \quad z=\zeta_{1}+z_{0} .
$$

and

Therefore

$$
\xi_{2}=\xi_{1} \cos \Delta \lambda-\eta \sin \Delta \lambda, \quad \eta_{2}=\xi_{1} \sin \Delta \lambda+\eta \cos \Delta \lambda,
$$

$$
\xi_{1}=\zeta \cos \phi+\xi \sin \phi, \quad \zeta_{1}=\zeta \sin \phi-\xi \cos \phi .
$$

$$
\begin{aligned}
& x=r_{0}+(\zeta \cos \phi+\xi \sin \phi) \cos \Delta \lambda-\eta \sin \Delta \lambda, \\
& y=\quad(\zeta \cos \phi+\xi \sin \phi) \sin \Delta \lambda+\eta \cos \Delta \lambda, \\
& z=z_{0}+\zeta \sin \phi-\xi \cos \phi .
\end{aligned}
$$

The inverse of this transformation is the transformation:

$$
\begin{aligned}
& \xi=\left[\left(x-r_{0}\right) \cos \Delta \lambda+y \sin \Delta \lambda\right] \sin \phi-\left(z-z_{0}\right) \cos \phi, \\
& \eta=-\left(x-r_{0}\right) \sin \Delta \lambda+y \cos \Delta \lambda, \\
& \zeta=\left[\left(x-r_{0}\right) \cos \Delta \lambda+y \sin \Delta \lambda\right] \cos \phi+\left(z-z_{0}\right) \sin \phi .
\end{aligned}
$$

If now we subject the system of differential equations (2) to the transformation (4), we obtain the system of differential equations,*

*The details of this process are the following:

becomes

$$
\frac{d^{2} x}{d t^{2}}-2 \omega \frac{d y}{d t}=\frac{\partial W}{\partial x}
$$

$$
\begin{gathered}
\cos \Delta \lambda\left(\frac{d^{2} \zeta}{d t^{2}} \cos \phi+\frac{d^{2} \xi}{d t^{2}} \sin \phi\right)-\sin \Delta \lambda \frac{d^{2} \eta}{d t^{2}}-2 \sin \Delta \lambda\left(\frac{d \zeta}{d t} \cos \phi+\frac{d \xi}{d t} \sin \phi\right) \omega-2 \cos \Delta \lambda \cdot \frac{d \eta}{d t} \cdot \omega \\
=\frac{\partial W}{\partial \xi} \sin \phi \cos \Delta \lambda-\frac{\partial W}{\partial \eta} \sin \Delta \lambda+\frac{\partial W}{\partial \zeta} \cos \phi \cos \Delta \lambda \\
\frac{d^{2} y}{d t^{2}}+2 \omega \frac{d x}{d t}=\frac{\partial W}{\partial y}
\end{gathered}
$$

becomes

$$
\begin{gathered}
\sin \Delta \lambda\left(\frac{d^{2} \zeta}{d t^{2}} \cos \phi+\frac{d^{2} \xi}{d t^{2}} \sin \phi\right)+\cos \Delta \lambda \frac{d^{2} \eta}{d t^{2}}+2 \cos \Delta \lambda\left(\frac{d \zeta}{d t} \cos \phi+\frac{d \xi}{d t} \sin \phi\right) \omega-2 \sin \Delta \lambda \frac{d \eta}{d t} \omega \\
=\frac{\partial W}{\partial \xi} \sin \phi \sin \Delta \lambda+\frac{\partial W}{\partial \eta} \cos \Delta \lambda+\frac{\partial W}{\partial \zeta} \cos \phi \sin \Delta \lambda . \\
\frac{d^{2} z}{d t^{2}}=\frac{\partial W}{\partial z}
\end{gathered}
$$

becomes

$$
\frac{\partial^{2} \zeta}{d t^{2}} \sin \phi-\frac{d^{2} \xi}{d t^{2}} \cos \phi=-\frac{\partial W}{\partial \xi} \cos \phi+\frac{\partial W}{\partial \zeta} \sin \phi .
$$

If we multiplỳ these equations respectively by $\cos \Delta \lambda \sin \phi, \sin \Delta \lambda \sin \phi,-\cos \phi$ and add, we obtain the first of the equations (5); if we multiply respectively by $\cos \Delta \lambda \cos \phi, \sin \Delta \lambda^{\prime}$ $\cos \phi, \sin \phi$, and add, we obtain the second of the equations (5); and if we multiply respectively by $-\sin \Delta \lambda, \cos \Delta \lambda, 0$, we obtain the last of the equations (5). 


$$
\begin{aligned}
& \frac{d^{2} \xi}{d t^{2}}-2 \omega \sin \phi \cdot \frac{d \eta}{d t}=\begin{array}{c}
\partial W \\
\partial \xi
\end{array} \\
& \frac{d^{2} \eta}{d t^{2}}+2 \omega\left(\sin \phi \cdot \frac{d \xi}{d t}+\cos \phi \cdot \frac{d \zeta}{d t}\right)=\frac{\partial W}{\partial \eta} \\
& \frac{d^{2} \zeta}{d t^{2}}-2 \omega \cos \phi \cdot \frac{d \eta}{d t}=\frac{\partial W}{\partial \zeta} .
\end{aligned}
$$

The initial conditions (3) assume the form:

(6) When $\quad \dot{t}=0, \quad \xi=\eta=\zeta=0, \quad \frac{d \xi}{d t}=\frac{d \eta}{d t}=\frac{d \zeta}{d t}=0$.

Therefore the curve $c$ is that solution of the differential equations (5) which is subject to the initial conditions (6).

\$4. Another derivation of equations (5). The differential equations (5) may also be obtained as follows. The projections on the moving axes $\eta, \xi, \zeta$ of the absolute velocity $V_{a}$ are *

$$
\begin{aligned}
& V_{a_{\eta}}=\frac{d \eta}{d t}+V_{\eta}^{0}+q \zeta-r \xi, \\
& V_{a \xi}=\frac{d \xi}{d t}+V_{\xi}^{0}+r \eta-p \zeta, \\
& V_{a \zeta}=\frac{d \zeta}{d t}+V_{\zeta}^{0}+p \xi-q \eta,
\end{aligned}
$$

where $V_{\eta}^{0}, V_{\xi}^{0}, V_{\xi}^{0}$ are the projections on the axes $\eta, \xi, \xi$ of the absolute velocity $V^{0}$ of the initial point $P_{0}$, and $p, q, r$ are the projections on the same axes of the rotation $\omega$ of the earth. From Fig. $2^{\prime}$ it is easily seen that

where

$$
V_{\eta}^{0}=V^{0} \cos \Delta \lambda, V_{\xi}^{0}=V^{0} \sin \Delta \lambda \sin \phi, V_{\zeta}^{0}=V^{0} \sin \Delta \lambda \cos \phi,
$$

and

$$
V^{0}=\omega r_{0},
$$

Therefore

$$
p=0, \quad q=\omega \cos \phi, \quad r=-\omega \sin \phi .
$$

$$
\begin{aligned}
& V_{a \eta}=\frac{d \eta}{d t}+\omega r_{0} \cos \Delta \lambda+\omega \cos \phi \cdot \zeta+\omega \sin \phi \cdot \xi, \\
& V_{a \xi}=\frac{d \xi}{d t}+\omega r_{0} \sin \Delta \lambda \sin \phi-\omega \sin \phi \cdot \eta, \\
& V_{a \zeta}=\frac{d \zeta}{d t}+\omega r_{0} \sin \Delta \lambda \cos \phi-\omega \cos \phi \cdot \eta .
\end{aligned}
$$

* See Appenr, Traité de Mécanique Rationnelle, vol. 1, (1902) \$61. 
The projections on the moving axes $\eta, \xi, \zeta$ of the absolute acceleration $J_{a}$ are

$$
\begin{aligned}
& J_{a \eta}=\frac{d V_{a \eta}}{d t}+q V_{a \zeta}-r V_{a \xi}, \\
& J_{a \xi}=\frac{d V_{a \xi}}{d t}+r V_{a_{\eta}}-p V_{a \zeta}, \\
& J_{a \zeta}=\frac{d V_{a \zeta}}{d t}+p V_{a \xi}-q V_{a \eta} .
\end{aligned}
$$

Hence by the expressions for $V_{a_{\eta}}, V_{a_{\xi}}, V_{a \zeta}$,

$$
J_{a_{\eta}}=\frac{d^{2} \eta}{d t^{2}}+2 \omega\left(\cos \phi \cdot \frac{d \zeta}{d t}+\sin \phi \frac{d \xi}{d t}\right)-\omega^{2}\left(\eta-r_{0} \sin \Delta \lambda\right),
$$

(8) $J_{a \xi}=\frac{d^{2} \xi}{d t^{2}}-2 \omega \sin \phi \cdot \frac{d \eta}{d t}-\omega^{2} \sin \phi\left(\cos \phi \cdot \zeta+\sin \phi \cdot \xi+r_{0} \cos \Delta \lambda\right)$,

$$
J_{a \zeta}=\frac{d^{2} \zeta}{d t^{2}}-2 \omega \cos \phi \cdot \frac{d \eta}{d t}-\omega^{2} \cos \phi\left(\cos \phi \cdot \zeta+\sin \phi \cdot \xi+r_{0} \cos \Delta \lambda\right) .
$$

Since gravitation acts instantaneously, the projections of the absolute force on the axes $\eta, \xi, \zeta$ are

$$
\frac{\partial U}{\partial \eta}, \quad \frac{\partial U}{\partial \xi}, \cdot \frac{\partial U}{\partial \zeta} .
$$

Hence the differential equations of motion are

$$
J_{a_{\eta}}=\frac{\partial U}{\partial \eta}, \quad J_{a \xi}=\frac{\partial U}{\partial \xi}, \quad \dot{J_{a \zeta}}=\frac{\partial U}{\partial \zeta},
$$

where the expressions for $J_{a_{\eta}}, J_{a \xi}, J_{a \zeta}$ are given by (8). In virtue of relations (1) and (4) we find that

$$
\begin{aligned}
& \frac{\partial W}{\partial \eta}=\frac{\partial U}{\partial \eta}+\omega^{2}\left(\eta-r_{0} \sin \Delta \lambda\right), \\
& \frac{\partial W}{\partial \xi}=\frac{\partial U}{\partial \xi}+\omega^{2} \sin \phi\left(\cos \phi \cdot \zeta+\sin \phi \cdot \xi+r_{0} \cos \Delta \lambda\right), \\
& \frac{\partial W}{d \zeta}=\frac{\partial U}{\partial \zeta}+\omega^{2} \cos \phi\left(\cos \phi \cdot \zeta+\sin \phi \cdot \xi+r_{0} \cos \Delta \lambda\right),
\end{aligned}
$$

and hence equations (9) are identical with equations (5).

$\S 5$. Integration of the differential equations of motion (5). Let us write equations (5) in the following form:

$(\alpha)$

$$
\begin{array}{lr}
\eta^{\prime \prime}+2 \omega\left(\sin \phi \cdot \xi^{\prime}+\cos \phi \cdot \zeta^{\prime}\right)-W_{\eta}=0, \\
\xi^{\prime \prime}-2 \omega \sin \phi \cdot \eta^{\prime} & -W_{\xi}=0, \\
\zeta^{\prime \prime}-2 \omega \cos \phi \cdot \eta^{\prime} & -W_{\zeta}=0,
\end{array}
$$


where the primes (') and seconds (') denote the first and second derivatives, with respect to the time $t$, of the functions to which they are attached, and the subscripts $(\eta, \xi, \varsigma)$ denote the first partial derivatives of $W$ with respect to the attached subscript. In the equations which follow thirds $\left({ }^{\prime \prime \prime}\right)$, etc., and double subscript $(\eta n, \eta \xi \cdots)_{0}$ etc., will be used to denote the higher derivatives. For the particular values of $\eta, \xi, \zeta$, and their derivatives with respect to $t$, which correspond to $t=0$, we shall use the symbols $\eta_{0}, \xi_{0}, \zeta_{0}, \eta_{0}^{\prime}, \xi_{0}^{\prime}, \zeta_{0}^{\prime}$, $\eta_{0}^{\prime \prime}, \xi_{0}^{\prime \prime}$, etc. Hence condition (6) may be written in the following form:

$$
\eta_{0}=\xi_{0}=\zeta_{0}=0, \quad \eta_{0}^{\prime}=\xi_{0}^{\prime}=\zeta_{0}^{\prime}=0 .
$$

For the particular values of the derivatives of $W$ which correspond to $\eta=\eta_{0}$, $\xi=\xi_{0}, \zeta=\zeta_{0}$, we shall use the symbols $W_{\eta}^{0}, W_{\xi}^{0}, W_{\zeta}^{0}, W_{\eta \eta}^{0}, W_{\eta \xi}^{0}, W_{\eta \zeta}^{0}$, $W_{\xi \xi}^{0}, W_{\xi \zeta}^{0}, W_{\zeta \zeta}^{0}$, etc.

For a set of functions

$$
\eta=\lambda(t), \quad \xi=\mu(t), \quad \zeta=\nu(t),
$$

which is a solution of the set of differential equations $(\alpha)$, the left hand member of each of the equations $(\alpha)$ vanishes identically. Therefore, for this solution, the derivative with respect to $t$ of each of these left-hand members must vanish identically. Hence the identities:

$$
\eta^{\prime \prime \prime}+2 \omega\left(\sin \phi \cdot \xi^{\prime \prime}+\cos \phi \cdot \zeta^{\prime \prime}\right)-\left[W_{\eta \eta} \cdot \eta^{\prime}+W_{\eta \xi} \cdot \xi^{\prime}+W_{\eta \zeta} \cdot \zeta^{\prime}\right]=0,
$$

$$
\begin{array}{ll}
\xi^{\prime \prime \prime}-2 \omega \sin \phi \cdot \eta^{\prime \prime} & -\left[W_{\xi \eta} \cdot \eta^{\prime}+W_{\xi \xi} \cdot \xi^{\prime}+W_{\xi \zeta} \cdot \zeta^{\prime}\right]=0, \\
\zeta^{\prime \prime \prime}-2 \omega \cos \phi \cdot \eta^{\prime \prime} & -\left[W_{\zeta \eta} \cdot \eta^{\prime}+W_{\zeta \xi} \cdot \xi^{\prime}+W_{\zeta \zeta} \cdot \zeta^{\prime}\right]=0 .
\end{array}
$$

Similarly, if we assume the existence and continuity of all the derivatives which are needed, we obtain the following sets of identities:

$$
\begin{aligned}
\eta^{\text {iv }} & +2 \omega\left(\sin \phi \cdot \xi^{\prime \prime \prime}+\cos \phi \cdot \zeta^{\prime \prime \prime}\right) \\
& -\left[W_{\eta \eta} \cdot \eta^{\prime \prime}+W_{\eta \xi} \cdot \xi^{\prime \prime}+W_{\eta \zeta} \zeta^{\prime \prime}+\alpha_{1} \eta^{\prime}+\beta_{1} \xi^{\prime}+\gamma_{1} \zeta^{\prime}\right]=0,
\end{aligned}
$$

$\left(\alpha^{\prime \prime}\right) \quad \xi^{\mathrm{iv}}-2 \omega \sin \phi \cdot \eta^{\prime \prime \prime}$

$$
\begin{aligned}
& -\left[W_{\xi \eta} \cdot \eta^{\prime \prime}+W_{\xi \xi} \cdot \xi^{\prime \prime}+W_{\xi \zeta} \cdot \zeta^{\prime \prime}+\alpha_{2} \eta^{\prime}+\beta_{2} \xi^{\prime}+\gamma_{2} \zeta^{\prime}\right]=0, \\
& \zeta^{\mathrm{iv}}-2 \omega \cos \phi \cdot \eta^{\prime \prime \prime} \\
& -\left[W_{\zeta \eta} \cdot \eta^{\prime \prime}+W_{\zeta \xi} \cdot \xi^{\prime \prime}+W_{\zeta \zeta} \cdot \zeta^{\prime \prime}+\alpha_{3} \eta^{\prime}+\beta_{3} \xi^{\prime}+\gamma_{3} \zeta^{\prime}\right]=0,
\end{aligned}
$$

where $\alpha_{i}, \beta_{i}, \gamma_{i}, i=1,2,3$, stand for homogeneous linear expressions in $\eta^{\prime}, \xi^{\prime}, \zeta^{\prime}$, the coefficients of which are third derivatives of $W^{*}$,

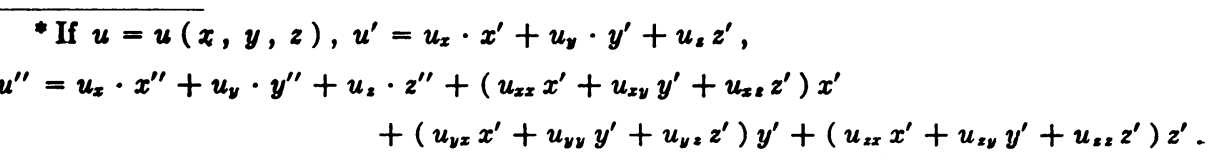




$$
\begin{aligned}
\eta^{\mathrm{v}} & +2 \omega\left(\sin \phi \cdot \xi^{\mathrm{iv}}+\cos \phi \cdot \zeta^{\mathrm{iv}}\right) \\
& -\left[W_{\eta \eta} \cdot \eta^{\prime \prime \prime}+W_{\eta \xi} \cdot \xi^{\prime \prime \prime}+W_{\eta \zeta} \cdot \zeta^{\prime \prime \prime}+\alpha_{1}^{\prime} \eta^{\prime}+\beta_{1}^{\prime} \xi^{\prime}+\gamma_{1}^{\prime} \zeta^{\prime}\right]=0,
\end{aligned}
$$

$\left(\alpha^{\prime \prime \prime}\right) \quad \xi v-2 \omega \sin \phi \cdot \eta^{\text {iv }}$

$$
\begin{aligned}
& -\left[W_{\xi \eta} \cdot \eta^{\prime \prime \prime}+W_{\xi \xi} \cdot \xi^{\prime \prime \prime}+W_{\xi \zeta} \cdot \zeta^{\prime \prime \prime}+\alpha_{2}^{\prime} \cdot \eta^{\prime}+\beta_{2}^{\prime} \xi^{\prime}+\gamma_{2}^{\prime} \zeta^{\prime}\right]=0, \\
\zeta^{\mathrm{v}} & -2 \omega \cos \phi \cdot \eta^{\mathrm{iv}} \\
& -\left[W_{\zeta \eta} \cdot \eta^{\prime \prime \prime}+W_{\zeta \xi} \cdot \xi^{\prime \prime \prime}+W_{\zeta \zeta} \cdot \zeta^{\prime \prime \prime}+\alpha_{3}^{\prime} \eta^{\prime}+\beta_{3}^{\prime} \xi^{\prime}+\gamma_{3}^{\prime} \zeta^{\prime}\right]=0,
\end{aligned}
$$

where $\alpha_{i}^{\prime}, \beta_{i}^{\prime}, \gamma_{i}^{\prime}, i=1,2,3$, stand for polynomials in the $\eta^{\prime}, \xi^{\prime}, \zeta^{\prime}, \eta^{\prime \prime}, \xi^{\prime \prime}, \zeta^{\prime \prime}$ and the third and fourth derivatives of $W .^{*}$

By condition $(\beta)$ we already know that

$$
\eta_{0}=\xi_{0}=\zeta_{0}=0, \quad \eta_{0}^{\prime}=\xi_{0}^{\prime}=\zeta_{0}^{\prime}=0 .
$$

We also know that

$$
W_{\eta}^{0}=W_{\xi}^{0}=0 \text {. }
$$

Therefore, when $t=0$, the identities $(\alpha)$ yield the relations

$$
\eta_{0}^{\prime \prime}=0, \quad \xi_{0}^{\prime \prime}=0, \quad \zeta_{0}^{\prime \prime}=W_{\zeta}^{0} ;
$$

the identities $\left(\alpha^{\prime}\right)$, the relations

$$
\eta_{0}^{\prime \prime \prime}=-2 \omega \cos \phi \cdot W_{\zeta}^{0}, \quad \xi_{0}^{\prime \prime \prime}=0, \quad \zeta_{0}^{\prime \prime \prime}=0 ;
$$

the identities $\left(\alpha^{\prime \prime}\right)$, the relations

$$
\begin{gathered}
\eta_{0}^{\text {iv }}=W_{\zeta}^{0} W_{\eta \zeta}^{0}, \quad \xi_{0}^{i v}=W_{\zeta}^{0}\left(W_{\xi \zeta}^{0}-4 \omega^{2} \sin \phi \cos \phi\right), \\
\zeta_{0}^{\text {iv }}=W_{\zeta}^{0}\left(W_{\zeta \zeta}^{0}-4 \omega^{2} \cos ^{2} \phi\right) ;
\end{gathered}
$$

and the identities $\left(\alpha^{\prime \prime \prime}\right)$, the relations

$$
\begin{aligned}
& \eta_{0}^{\nabla}=-2 \omega W_{\zeta}^{0}\left[\sin \phi \cdot W_{\xi \zeta}^{0}+\cos \phi\left(W_{\zeta \zeta}^{0}+W_{\eta \eta}^{0}-4 \omega^{2}\right)\right], \\
& \xi_{0}^{\nabla}=+2 \omega W_{\zeta}^{0}\left[\sin \phi \cdot W_{\eta \zeta}^{0}-\cos \phi \cdot W_{\xi \eta}^{0}\right] \text {, } \\
& \zeta_{0}^{\nabla}=+2 \omega W_{\zeta}^{0} \cos \phi\left[W_{\eta \zeta}^{0}-W_{\zeta \eta}^{0}\right]=0 \text {. } \\
& u^{\prime \prime \prime}=u_{x} x^{\prime \prime \prime}+u_{y} y^{\prime \prime \prime}+u_{z} z^{\prime \prime \prime}+3\left[\left(u_{x x} x^{\prime \prime}+u_{x y} y^{\prime \prime}+u_{x} z^{\prime \prime}\right) x^{\prime}\right. \\
& \left.+\left(u_{x y} x^{\prime \prime}+u_{y y} y^{\prime \prime}+u_{y z} z^{\prime \prime}\right) y^{\prime}+\left(u_{z x} x^{\prime \prime}+u_{z y} y^{\prime \prime}+u_{z z} z^{\prime \prime}\right) z^{\prime}\right] \\
& +\left\{\begin{array}{c}
\left(u_{z x x} x^{\prime}+u_{z x y} y^{\prime}+u_{x z s} z^{\prime}\right) x^{\prime} \\
+\left(u_{x y z} x^{\prime}+u_{x y y} y^{\prime}+u_{x y z} z^{\prime}\right) y^{\prime} \\
+\left(u_{z z x} x^{\prime}+u_{x z y} y^{\prime}+u_{x z z} z^{\prime}\right) z^{\prime}
\end{array}\right\} x^{\prime}+\left\{\begin{array}{r}
\left(u_{y z z} x^{\prime}+u_{y z y} y^{\prime}+u_{y z z} z^{\prime}\right) x^{\prime} \\
+\left(u_{y y z} x^{\prime}+u_{y y y} y^{\prime}+u_{y y z} z^{\prime}\right) y^{\prime} \\
+\left(u_{y z z} x^{\prime}+u_{y z y} y^{\prime}+u_{y z z} z^{\prime}\right) z^{\prime}
\end{array}\right\} y^{\prime} \\
& +\left\{\begin{array}{r}
\left(u_{z x x} x^{\prime}+u_{z y} y^{\prime}+u_{z x z} z^{\prime}\right) x^{\prime} \\
+\left(u_{s y x} x^{\prime}+u_{s y y} y^{\prime}+u_{z y z} z^{\prime}\right) y^{\prime} \\
+\left(u_{s z x} x^{\prime}+u_{s z y} ;-u_{z z s} z^{\prime}\right) z^{\prime}
\end{array}\right\} z^{\prime}
\end{aligned}
$$


Let us now assume that the conditions are satisfied under which the set of differential equations (5) or $(\alpha)$ have a solution of the form

$$
\begin{aligned}
& \eta=a_{0}+a_{1} t+a_{2} t^{2}+a_{3} t^{3}+a_{4} t^{4}+a_{5} t^{5}+\cdots, \\
& \xi=b_{0}+b_{1} t+b_{2} t^{2}+b_{3} t^{3}+b_{4} t^{4}+b_{5} t^{5}+\cdots, \\
& \zeta=c_{0}+c_{1} t+c_{2} t^{2}+c_{3} t^{3}+c_{4} t^{4}+c_{5} t^{5}+\cdots,
\end{aligned}
$$

in the neighborhood of the point $P_{0}$. It then follows, from the preceding work, that for the initial conditions $(6)$ or $(\beta)$,

$$
\begin{aligned}
& a_{0}=\eta_{0}=0, \quad a_{1}=\eta_{0}^{\prime}=0, \quad a_{2}=\frac{1}{2} \eta_{0}^{\prime \prime}=0, \\
& b_{0}=\xi_{0}=0, \quad b_{1}=\xi_{0}^{\prime}=0, \quad b_{2}=\frac{1}{2} \xi_{0}^{\prime \prime}=0, \\
& c_{0}=\zeta_{0}=0, \quad c_{1}=\zeta_{0}^{\prime}=0, \quad c_{2}=\frac{1}{2} \zeta_{0}^{\prime \prime}=\frac{1}{2} W_{\zeta}^{0} ; \\
& a_{3}=\frac{1}{6} \eta_{0}^{\prime \prime \prime}=-\frac{1}{3} \omega \cos \phi \cdot W_{\zeta}^{0}, \\
& b_{3}=\frac{1}{6} \xi_{0}^{\prime \prime \prime}=0, \\
& c_{3}=\frac{1}{6} \zeta_{0}^{\prime \prime \prime}=0 ; \\
& a_{4}=\frac{1}{24} \eta_{0}^{\mathrm{iv}}=\frac{1}{24} W_{\zeta}^{0} W_{\eta \zeta}^{0}, \\
& b_{4}=\frac{1}{24} \xi_{0}^{\mathrm{iv}}=\frac{1}{24} W_{\zeta}^{0}\left(W_{\xi \zeta}^{0}-4 \omega^{2} \sin \phi \cos \phi\right), \\
& c_{4}=\frac{1}{24} \zeta_{0}^{\mathrm{iv}}=\frac{1}{24} W_{\zeta}^{0}\left(W_{\zeta \zeta}^{0}-4 \omega^{2} \cos { }^{2} \phi\right) ; \\
& a_{5}=\frac{1}{12} \delta \eta_{0}^{\mathrm{v}}=-\frac{1}{6} \sigma \omega W_{\zeta}^{0}\left[\sin \phi \cdot W_{\xi \zeta}^{0}+\cos \phi\left(W_{\zeta \zeta}^{0}+W_{\eta \eta}^{0}-4 \omega^{2}\right)\right], \\
& b_{5}=\frac{1}{1} \delta \xi_{0}^{\mathrm{v}}=\frac{1}{6} \sigma \omega W_{\zeta}^{0}\left[\sin \phi \cdot W_{\eta \zeta}^{0}-\cos \phi W_{\xi \eta}^{0}\right], \\
& c_{5}=\frac{1}{1} \frac{1}{2} \delta \zeta_{0}^{\mathrm{v}}=0 .
\end{aligned}
$$

The equations (10), for which the constants are given by the relations (11), are then the equations of the curve $c$ referred to the cardinal axes at $P_{0}$.

\$6. The plumb-bob locus referred to the cardinal axes of $P_{0}$. This curve has already been defined as the locus of plumb-bobs of all plumb-lines which are supported at the initial point $P_{0}$, of the falling particle. It is therefore the locus of the feet of perpendiculars dropped from $P_{0}(\eta=0, \xi=0, \zeta=0)$ to the level (equipotential) surfaces $W=$ const. Its equations are easily seen to be

$$
\frac{\eta}{\frac{\partial W}{\partial \eta}}=\frac{\xi}{\frac{\partial W}{\partial \xi}}=\frac{\zeta}{\frac{\partial W}{\partial \zeta}} .
$$

Now let us solve these equations in the form

$$
\eta=\alpha_{1} \zeta+\alpha_{2} \zeta^{2}+\cdots, \quad \xi=\beta_{1} \zeta+\beta_{2} \zeta^{2}+\cdots .
$$


In order to do this let us write the equations (12) in the form:

where

$$
F_{i}(\eta, \xi, \zeta)=0 \text {, }
$$

$$
F_{1}=\left|\begin{array}{cc}
\xi & \zeta \\
W_{\xi} & W_{\zeta}
\end{array}\right| \quad \text { and } \quad F_{2}=\left|\begin{array}{cc}
\zeta & \eta \\
W_{\zeta} & W_{\eta}
\end{array}\right| \text {. }
$$

The derivatives $d \eta / d \zeta, d \xi / d \zeta, d^{2} \eta / d \zeta^{2}, d^{2} \xi / d \zeta^{2}$ are expressible in terms of the derivatives of $F_{i}, i=1,2,{ }^{*}$ and the derivatives of $F_{i}$ are expressible in terms of the derivatives of $W . \dagger$ The coefficients $\alpha_{1}, \alpha_{2}, \beta_{1}, \beta_{2}$ have then the following values:

$$
\begin{gathered}
\alpha_{1}=\left(\frac{d \eta}{d \zeta}\right)_{0}=0, \quad \beta_{1}=\left(\frac{d \xi}{d \zeta}\right)_{0}=0, \\
\alpha_{2}=\frac{1}{2}\left(\frac{d^{2} \eta}{d \zeta^{2}}\right)_{0}=\frac{W_{\eta \zeta}^{0}}{W_{\zeta}^{0}}, \quad \beta_{2}=\frac{W_{\xi \zeta}^{0}}{W_{\zeta}^{0}} .
\end{gathered}
$$

Therefore the plumb-bob locus may be represented by the equations

$$
\eta=\frac{W_{\eta \zeta}^{0}}{W_{\zeta}^{0}} \zeta^{2}+\cdots, \quad \xi=\frac{W_{\xi \xi}^{0}}{W_{\zeta}^{0}} \zeta^{2}+\cdots .
$$

\$7. The curve $c$ referred to axes of origin $P_{0}$ and directions which are parallel to the cardinal directions at the plumb-bob of a plumb-line which is supported at $P_{0}$. Let us denote by $R$ (Fig. 3 ) the point at which the plumb-bob of a

* The relations are the following:

$$
\begin{array}{cc}
\frac{\partial F_{i}}{\partial \eta} \frac{d \eta}{d \zeta}+\frac{\partial F_{i}}{\partial \xi} \frac{d \xi}{d \zeta}+\frac{\partial F_{i}}{\partial \zeta}=0 & (i=1,2), \\
\frac{\partial F_{i}}{\partial \eta} \frac{d^{2} \eta}{d \zeta^{2}}+\frac{d \eta}{d \zeta}\left[\frac{\partial^{2} F_{i}}{\partial \eta \partial \xi} \frac{d \xi}{d \zeta}+\right. & \left.\frac{\partial^{2} F_{i}}{\partial \eta^{2}} \frac{d \eta}{d \zeta}+\frac{\partial^{2} F_{i}}{\partial \zeta \partial \eta}\right]+\frac{\partial^{2} F_{i}}{\partial \zeta \partial \eta} \frac{d \eta}{d \zeta}+\frac{\partial^{2} F_{i}}{\partial \zeta^{2}}+\frac{\partial F_{i}}{\partial \xi} \frac{d^{2} \xi}{d \zeta^{2}} \\
+\frac{d \xi}{d \zeta}\left[\frac{\partial^{2} F_{i}}{\partial \xi^{2}} \frac{d \xi}{\partial \zeta}+\frac{\partial^{2} F_{i}}{d \xi \partial \eta} \frac{d \eta}{d \zeta}+\frac{\partial^{2} F_{i}}{\partial \zeta \partial \xi}\right]+\frac{\partial^{2} F_{i}}{\partial \zeta \partial \xi} \frac{d \xi}{d \zeta}=0 & (i=1,2) .
\end{array}
$$

$\dagger$ The relations which are needed are the following:

$$
\begin{aligned}
& \frac{\partial F_{1}}{\partial \eta}=\left|\begin{array}{cc}
\xi & \zeta \\
W_{\xi \eta} & W_{\zeta \eta}
\end{array}\right|, \quad \frac{\partial F_{1}}{\partial \xi}=\left|\begin{array}{cc}
\xi & \zeta \\
W_{\xi \xi} & W_{\zeta \xi}
\end{array}\right|+\left|\begin{array}{cc}
1 & 0 \\
W_{\xi} & W_{\xi}
\end{array}\right|, \quad \frac{\partial F_{1}}{\partial \zeta}=\left|\begin{array}{cc}
\xi & \zeta \\
W_{\xi \xi} & W_{\zeta \zeta}
\end{array}\right|+\left|\begin{array}{cc}
0 & 1 \\
W_{\xi} & W_{\xi}
\end{array}\right|, \\
& \frac{\partial F_{2}}{\partial \eta}=\left|\begin{array}{cc}
\zeta & \eta \\
W_{\zeta \eta} & W_{\eta \eta}
\end{array}\right|+\left|\begin{array}{cc}
0 & 1 \\
W_{\zeta} & W_{\eta}
\end{array}\right|, \quad \frac{\partial F_{2}}{\partial \xi}=\left|\begin{array}{cc}
\zeta & \eta \\
W_{\zeta \xi} & W_{\eta \xi}
\end{array}, \quad \frac{\partial F_{2}}{\partial \zeta}=\right| \begin{array}{cc}
\zeta & \eta \\
W_{\zeta \zeta} & W_{\eta \zeta}
\end{array}|+| \begin{array}{cc}
1 & 0 \\
W_{\zeta} & W_{\eta}
\end{array} \mid, \\
& \frac{\partial^{2} F_{1}}{\partial \zeta^{2}}=\left|\begin{array}{cc}
\xi & \zeta \\
W_{\xi \zeta \zeta \zeta} & W_{\zeta \zeta \zeta}
\end{array}\right|+\left|\begin{array}{cc}
0 & 1 \\
W_{\xi \zeta} & W_{\zeta \zeta}
\end{array}\right|-W_{\xi \zeta,}, \\
& \frac{\partial^{2} F_{2}}{\partial \zeta^{2}}=\left|\begin{array}{cc}
\zeta & \eta \\
W_{\zeta \zeta S} & W_{\eta \zeta S}
\end{array}\right|+\left|\begin{array}{cc}
1 & 0 \\
W_{\zeta \zeta} & W_{\eta \zeta}
\end{array}\right|+W_{\eta \zeta} ;
\end{aligned}
$$

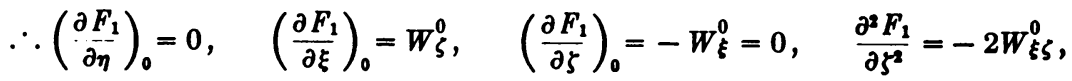

$$
\begin{aligned}
& \left(\frac{\partial F_{2}}{\partial \eta}\right)_{0}=-W_{\zeta}^{0}, \quad\left(\frac{\partial F_{2}}{\partial \xi}\right)_{0}=0, \quad\left(\frac{\partial F_{2}}{\partial \zeta}\right)_{0}=W_{\eta}^{0}=0, \quad \frac{\partial^{2} F_{2}}{\partial \zeta^{2}}=2 W_{\eta \zeta}^{0} .
\end{aligned}
$$


plumb-line which is supported at $P_{0}$ is in equilibrium under the tension of the plumb-line and the force of the field whose potential function is represented by $W$. The line $R P_{0}$ is then the vertical at $R$. Let us denote by $R E_{z}$ and $R S_{z}$ the easterly and the southerly directions at $R$. Now let us draw through $P_{0}$ the axes $P_{0} \bar{\eta}, P_{0} \bar{\xi}, P_{0} \bar{\zeta}$ parallel to $R E_{R}, R S_{R}$ and $R P_{0}$ respectively $\left(P_{0} \bar{\zeta}\right.$ is the continuation of the plumb-line $\left.R P_{0}\right)$. Let us denote by $\left(\alpha_{1}, \beta_{1}, \gamma_{1}\right)$, $\left(\alpha_{2}, \beta_{2}, \gamma_{2}\right)$ and $\left(\alpha_{3}, \beta_{3}, \gamma_{3}\right)$ the directional angles of the axes $P_{0}-\bar{\eta}, \bar{\xi}, \bar{\zeta}$ with respect to the cardinal axes $P_{0}-\eta, \xi, \zeta$. Then

$$
\begin{aligned}
& \bar{\eta}=\cos \alpha_{1} \cdot \eta+\cos \beta_{1} \cdot \xi+\cos \gamma_{1} \cdot \zeta, \\
& \bar{\xi}=\cos \alpha_{2} \cdot \eta+\cos \beta_{2} \cdot \xi+\cos \gamma_{2} \cdot \zeta, \\
& \bar{\zeta}=\cos \alpha_{3} \cdot \eta+\cos \beta_{3} \cdot \xi+\cos \gamma_{3} \cdot \zeta .
\end{aligned}
$$

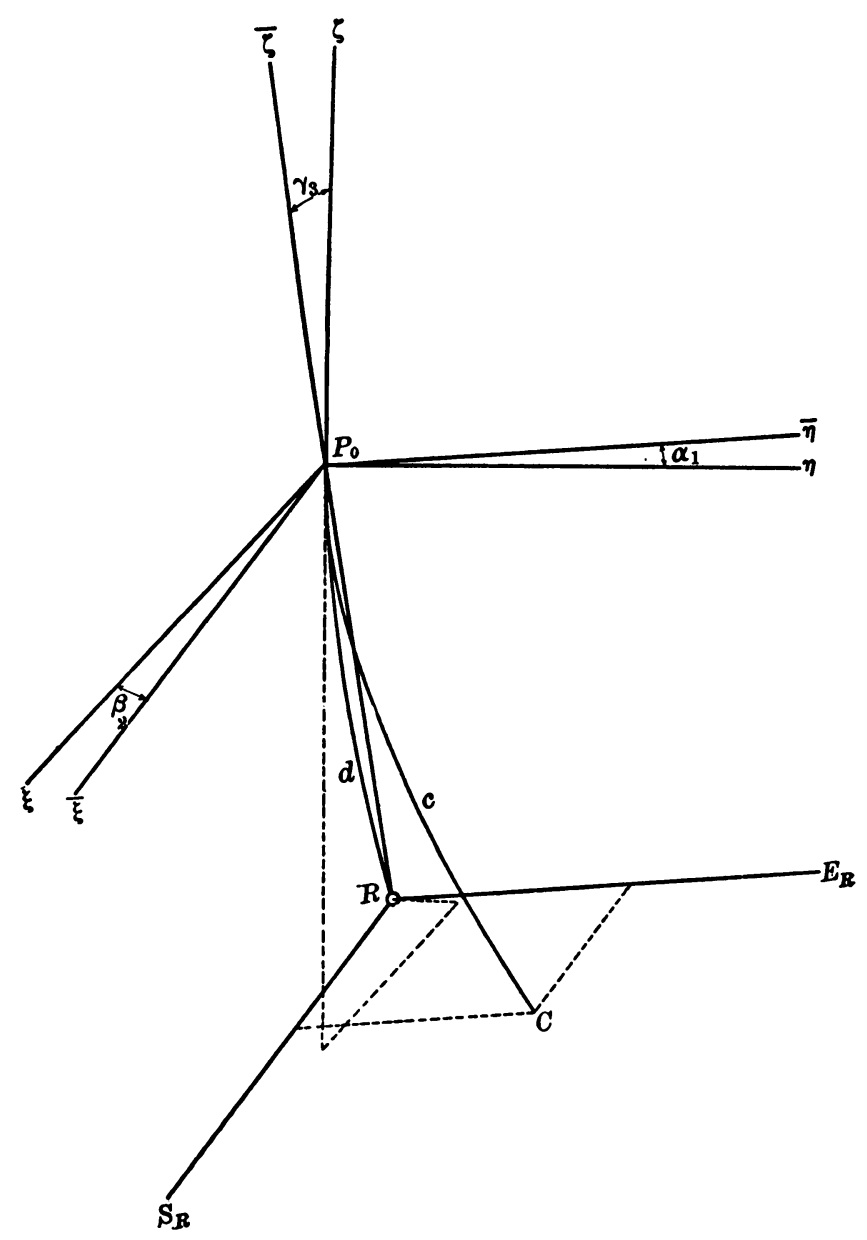

Fia. 3. 
In order to obtain expressions for these directional cosines let us recall that, at a general point $P,(1)$ the vertical is the normal to the level (equipotential) surface which passes through $P,(2)$ the east-and-west line is the intersection of the horizontal plane and the plane $(z=$ const.) which passes through $P$ and is perpendicular to the axis of rotation of the earth, (3) the north-and-south line is the common perpendicular to the vertical and the east-and-west line. Therefore, at a general point $P,(\eta, \xi, \zeta),(1)$ the directional cosines * of the vertical are proportional to

$$
W_{\eta}, W_{\xi}, W_{\zeta}
$$

(2) those of the east-and-west line are proportional to

where

$$
H, \text { 军, } Z \text {, }
$$

$$
\begin{gathered}
H=\left|\begin{array}{ll}
W_{\xi} & W_{\zeta} \\
\frac{\partial z}{\partial \xi} & \frac{\partial z}{\partial \zeta}
\end{array}\right|=\sin \phi \cdot W_{\xi}+\cos \phi \cdot W_{\zeta}, \\
\Xi=\left|\begin{array}{ll}
W_{\zeta} & W_{\eta} \\
\frac{\partial z}{\partial \zeta} & \frac{\partial z}{\partial \eta}
\end{array}\right|=-\sin \phi \cdot W_{\eta}, \\
Z Z=\left|\begin{array}{cc}
W_{\eta} & W_{\xi} \\
\frac{\partial z}{\partial \eta} & \frac{\partial z}{\partial \xi}
\end{array}\right|=-\cos \phi \cdot W_{\eta} ;
\end{gathered}
$$

and (3) those of the north-and-south line are proportional to

$$
\left|\begin{array}{cc}
W_{\xi} & W_{\zeta} \\
\Xi & Z
\end{array}\right|, \quad\left|\begin{array}{cc}
W_{\zeta} & W_{\eta} \\
Z & H
\end{array}\right|, \quad\left|\begin{array}{cc}
W_{\eta} & W_{\xi} \\
H & \Xi
\end{array}\right| .
$$

If the point $P$ is not too far from $P_{0}$, we may write without appreciable error,

$$
\begin{array}{ll}
W_{\eta}= & W_{\eta \eta}^{0} \cdot \eta+W_{\eta \xi}^{0} \cdot \xi+W_{\eta \zeta}^{0} \cdot \zeta, \\
W_{\xi}= & W_{\xi \eta}^{0} \cdot \eta+W_{\xi \xi}^{0} \cdot \xi+W_{\xi \zeta}^{0} \cdot \zeta, \\
W_{\zeta}=W_{\zeta}^{0}+W_{\zeta \eta}^{0} \cdot \eta+W_{\zeta \xi}^{0} \cdot \xi+W_{\zeta \zeta}^{0} \cdot \zeta .
\end{array}
$$

Therefore, for the point $R$, whose coordinates are

we may write

$$
\eta=\frac{W_{\eta \zeta}^{0}}{W_{\zeta}^{0}} \zeta^{2}, \quad \xi=\frac{W_{\xi \zeta}^{0}}{W_{\zeta}^{0}} \zeta^{2}, \quad \zeta=\zeta,
$$

$$
W_{\eta}^{R}=W_{\eta \zeta}^{0} \cdot \zeta, \quad W_{\xi}^{R}=W_{\xi \zeta}^{0} \cdot \zeta, \quad W_{\zeta}^{R}=W_{\zeta}^{0}+W_{\zeta \zeta}^{0} \cdot \zeta,
$$

- With respect to the cardinal directions of $P_{0}$. 
and hence

$$
\cos \alpha_{3}: \cos \beta_{3}: \cos \gamma_{3}=W_{\eta \zeta}^{0} \cdot \zeta: W_{\xi \zeta}^{0} \cdot \zeta: W_{\zeta}^{0}+W_{\zeta \zeta}^{0} \cdot \zeta,
$$

$\cos \alpha_{1}: \cos \beta_{1}: \cos \gamma_{1}$

$$
=\cos \phi \cdot W_{\zeta}^{0}+\left(\sin \phi \cdot W_{\xi \zeta}^{0}+\cos \phi \cdot W_{\zeta \zeta}^{0}\right) \zeta:-\sin \phi \cdot W_{\eta \zeta}^{0} \cdot \zeta:-\cos \phi \cdot W_{\eta \zeta}^{0} \cdot \zeta,
$$

$\cos \alpha_{2}: \cos \beta_{2}: \cos \gamma_{2}$

$$
\begin{aligned}
= & \sin \phi \cdot W_{\zeta}^{0} \cdot W_{\eta \zeta}^{0} \cdot \zeta: \cos \phi\left(W_{\zeta}^{0}\right)^{2} \\
& +W_{\zeta}^{0}\left(\sin \phi \cdot W_{\xi \zeta}^{0}+2 \cos \phi \cdot W_{\zeta \zeta}^{0}\right) \zeta:-\cos \phi \cdot W_{\zeta}^{0} \cdot W_{\xi \zeta}^{0} \cdot \zeta,
\end{aligned}
$$

for terms of order not greater than the first. In order to get the cosines themselves, we observe that *

$$
\begin{gathered}
\left(\left(W_{\eta \zeta}^{0} \cdot \zeta\right)^{2}+\left(W_{\xi \zeta}^{0} \cdot \zeta\right)^{2}+\left(W_{\zeta}^{0}+W_{\zeta \zeta}^{0} \zeta\right)^{2}\right)^{-1}=\frac{1}{W_{\xi}^{0}}\left(1-\frac{W_{\zeta \zeta}^{0}}{\left.W_{\zeta}^{0} \zeta+\cdots\right),}\right. \\
\left(\left\{\cos \phi \cdot W_{\zeta}^{0}+\left(\sin \phi \cdot W_{\xi \zeta}^{0}+\cos \phi \cdot W_{\zeta \zeta}^{0}\right) \zeta\right\}^{2}+\left(-\sin \phi \cdot W_{\eta \zeta}^{0} \cdot \zeta\right)^{2}\right. \\
\left.+\left(-\cos \phi \cdot W_{\eta \zeta}^{0} \cdot \zeta\right)^{2}\right)^{-1}=\frac{1}{\cos \phi \cdot W_{\zeta \zeta}^{0}}\left(1-\frac{\left.\sin \phi \cdot W_{\xi \zeta}^{0}+\cos \phi \cdot W_{\zeta \zeta}^{0} \zeta+\cdots\right),}{\cos \phi \cdot W_{\zeta}^{0}}\right. \\
\left(\left(\sin \phi \cdot W_{\zeta}^{0} \cdot W_{\eta \zeta}^{0} \cdot \zeta\right)^{2}+\left\{\cos \phi \cdot\left(W_{\zeta \zeta}^{0}\right)^{2}+W_{\zeta}^{0}\left(\sin \phi \cdot W_{\xi \zeta}^{0}\right.\right.\right. \\
\left.\left.\left.\quad+2 \cos \phi \cdot W_{\zeta \zeta}^{0}\right) \zeta\right\}^{2}+\left(-\cos \phi \cdot W_{\zeta}^{0} \cdot W_{\xi \zeta}^{0} \cdot \zeta\right)^{2}\right)^{-1} \\
=\frac{1}{\cos \phi \cdot\left(W_{\zeta}^{0}\right)^{2}}\left(1-\frac{\sin \phi \cdot W_{\xi \zeta}^{0}+2 \cos \phi \cdot W_{\zeta \zeta}^{0}}{\cos \phi \cdot W_{\zeta}^{0}}+\cdots\right) .
\end{gathered}
$$

Therefore

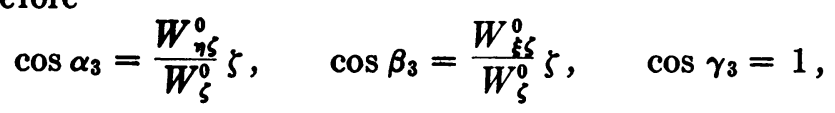

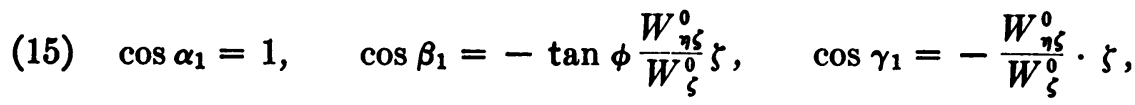

$$
\cos \alpha_{2}=\tan \phi \cdot \frac{W_{\eta \zeta}^{0}}{W_{\zeta}^{0}} \cdot \zeta, \quad \cos \beta_{2}=1, \quad \cos \gamma_{2}=-\frac{W_{\xi \zeta}^{0}}{W_{\zeta}^{0}} \cdot \zeta,
$$

for terms of order not higher than the first. In virtue of the values $(15)$, equations (14) assume the form:

$$
\begin{aligned}
& \bar{\eta}=\eta-\tan \phi \frac{W_{\eta \zeta}^{0}}{W_{\zeta}^{0}} \xi \zeta-\frac{W_{\eta \zeta}^{0}}{W_{\zeta}^{0}} \zeta^{2}, \\
& \xi=\tan \phi \cdot \frac{W_{\eta \zeta}^{0}}{W_{\zeta}^{0}} \eta \zeta+\xi-\frac{W_{\xi \zeta}^{0}}{W^{0}} \zeta^{2}, \\
& \bar{\zeta}=\frac{W^{0}}{W_{\zeta \zeta}^{0}} \eta \zeta+\frac{W_{\xi \zeta}^{0}}{W_{\zeta}^{0}} \xi \zeta+\zeta, \\
& \frac{1}{\sqrt{A+B \zeta+C \zeta^{2}+\cdots}}=\frac{1}{A^{\frac{1}{2}}}\left(1-\frac{1}{2} \frac{B}{A} \zeta+\frac{3 B^{2}-4 A C}{8 A^{2}} \zeta^{2}+\cdots\right) \text {. }
\end{aligned}
$$


in which we must now substitute for $\eta, \xi, \zeta$ the values in $t$ which are given by equations (10). Doing this, we find *

$$
\begin{array}{r}
\bar{\eta}=-\frac{1}{8} \omega \cos \phi \cdot W_{\zeta}^{0} \cdot t^{3}-\frac{\delta}{24} W_{\zeta}^{0} \cdot W_{\eta \zeta}^{0} \cdot t^{4}-\frac{1}{60} \omega W_{\zeta}^{0}\left(\sin \phi \cdot W_{\xi \zeta}^{0}\right. \\
\left.+\cos \phi\left(W_{\zeta \zeta}^{0}+W_{\eta}^{0 \eta}-4 \omega^{2}\right)\right) t^{5},
\end{array}
$$

(17) $\xi=-\left(\frac{1}{6} \omega^{2} \sin \phi \cos \phi+\frac{{ }_{2}^{5}}{4} W_{\xi \zeta}^{0}\right) W_{\zeta}^{0} \cdot t^{4}$

$$
\begin{array}{r}
-\frac{1}{60} \omega W_{\zeta}^{0}\left(9 \sin \phi W_{\eta \zeta}^{0}+\cos \phi W_{\xi \eta}^{0}\right) t^{5}, \\
\bar{\zeta}=\frac{1}{2} W_{\zeta}^{0} \cdot t^{2}+\frac{1}{24}\left(W_{\zeta \zeta}^{0}-4_{\iota} \omega^{2} \cos ^{2} \phi\right) W_{\zeta}^{0} \cdot t^{4}-\frac{1}{6} \omega \cos \phi W_{\zeta}^{0} W_{\eta \zeta}^{0} t^{5} .
\end{array}
$$

Equations (17) are the equations of the curve $c$ referred to the axes $P_{0}-\bar{\eta}, \bar{\xi}, \bar{\zeta}$.

If we put $-\bar{\zeta}=h=R P_{0}$, where $h$ is the height through which the particle falls, then the corresponding values of $\bar{\eta}$ and $\bar{\xi}$ are the easterly and the southerly deviations which correspond to $h$. These, and not the quantities $\eta, \xi$, are the ones which are measured in experiments.

WASHINGTON UNIVERSITY,

$$
\text { ST. Lours, Mo., April, } 1912 .
$$

* We can not carry these developments beyond $t^{5}$ unless we carry the developments (15) beyond $\zeta$. 